\title{
Validation of DeLone and McLean model to analyze decision support systems success in the banking sector of Oman
}

\author{
Anu Manchanda ${ }^{1}$,Dr. Saurabh Mukherjee ${ }^{2}$ \\ Waljat College of Applied Sciences,Muscat \\ anu@waljat.net \\ Associate Professor, Department of Computer Science,BanasthaliUniversity,Jaipur, India \\ saurabh.mukherjee@rediffmail.com
}

\section{ABSTRACT}

The main objective of this paper is to investigate empirically the validity of DeLone and McLean model in measuring the decision support system success in the banking sector of Oman. Data was collected from decision support system users working in the banks of Oman. Data analysis was done using Structural Equation Modelling (SEM) through smartPLS 2.0. The results of the study showed that system quality and information quality has no influence on system use, system quality and information quality has influence on user satisfaction, system use had no influence on user satisfaction, user satisfaction has no influence on system use, system use had no influence on individual impact, individual impact has no influence on system use, user satisfaction has influence on individual impact and individual impact has influence on user satisfaction.

\section{Indexing terms/Keywords}

DeLone and McLean model, decision support system, system quality, information quality, system use, user satisfaction, individual impact.

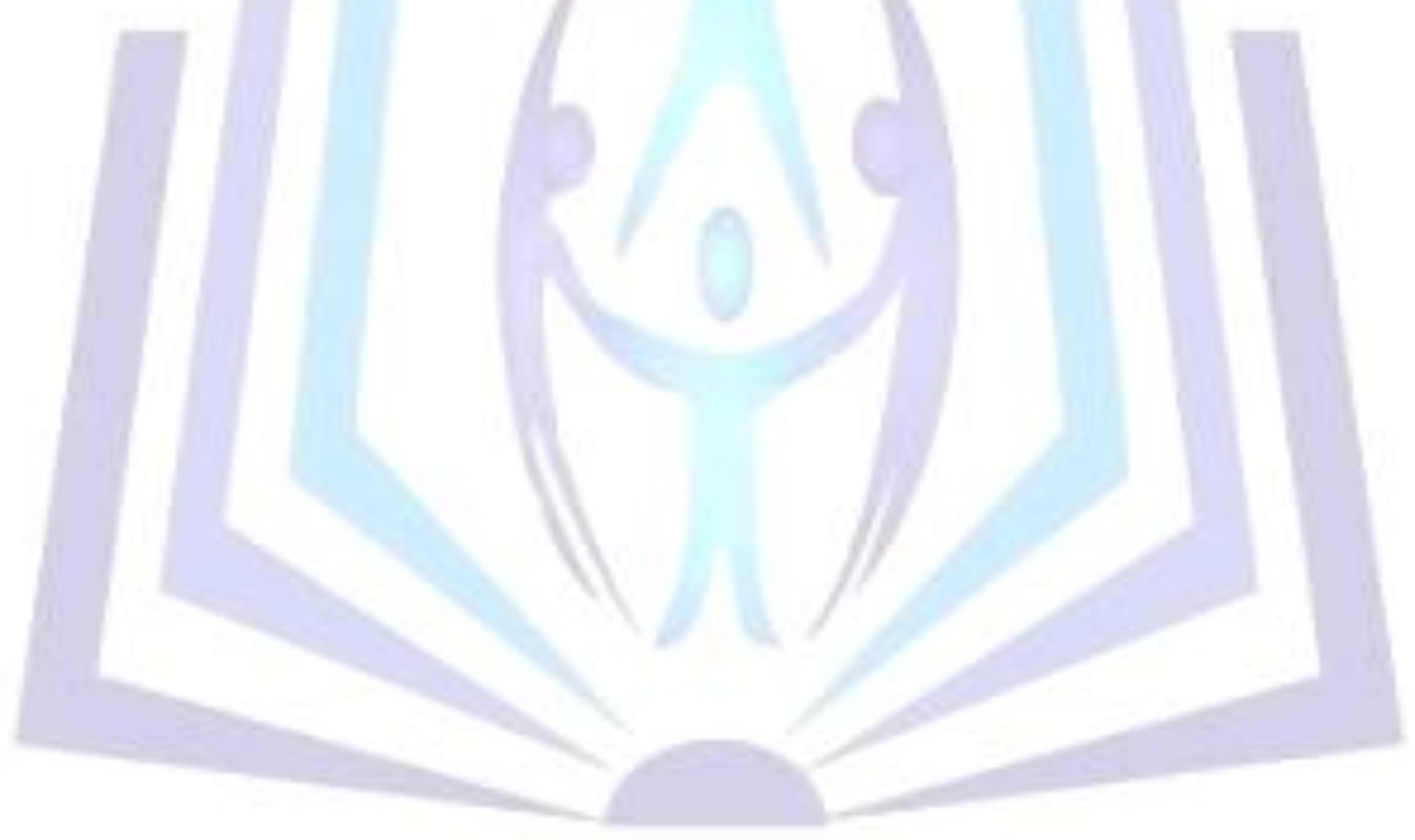

\section{Council for Innovative Research}

Peer Review Research Publishing System

Journal: INTERNATIONAL JOURNAL OF COMPUTERS \& TECHNOLOGY

Vol 13 ., No 12.

www.ijctonline.com , editorijctonline@gmail.com 


\section{INTRODUCTION}

Decision support systems (DSS) are special type of information systems capable to perform complex data analysis and to help decision makers to carry out decision making effectively. In banks managers need to deal with complex situations and take decisions based on the information retrieved after processing huge amount of data. Managers are always under stress and to remain competitive in the banking industry these decisions should be taken with utmost care. Decision support systems in the banks execute complex operations on the data according to the requirements of the users at a fast rate and generate results in useful formats. Banks are heavily investing in DSS as they need to do business in trading and investment in which the historical data is processed to obtain and generate the best outcomes for further decision making. There is rapid growth in the use of DSS in different financial sectors but there is still scarcity of models and lack of studies to evaluate these systems especially in Oman. This has led to carry out this study and suggest a model to analyze DSS success in the banking sector.

DeLone and McLean model (2003) of IS success has been the most popular model in the literature (Brown, 2008) and has received attention so this model is taken as a foundation model for research in DSS in banking sector. This studyempirically validates DeLone and McLean model of IS success.

\section{LITERATURE REVIEW}

\subsection{Decision support systems}

Decision support systems are specialized information systems. Various definitions of DSS can be given based upon their functions.(Little,1970) defined DSS as "model-based set of procedures for processing data and judgments to assist a manager in his decision making". The main characteristics of DSS include facilitation in decision making process, supporting the decision making and quick response according to change in requirements. Different terms are used for specific types of DSS like business intelligence etc.

\subsection{DeLone and McLean model of IS success}

Before the development of DeLone and McLean model research in the field of information system success was not consistent and all the aspects of success were not organized properly. After comprehensive evaluation of the literature in year 1992 DeLone and McLean presented a model of IS success. The DeLone\& McLean's IS Success Model (1992) identified factors leading to information system success. They suggested six main constructs of IS success namely system quality, information quality, use, user satisfaction, individual impact, organization impact. The figure 1 presented below depicts the IS success model.

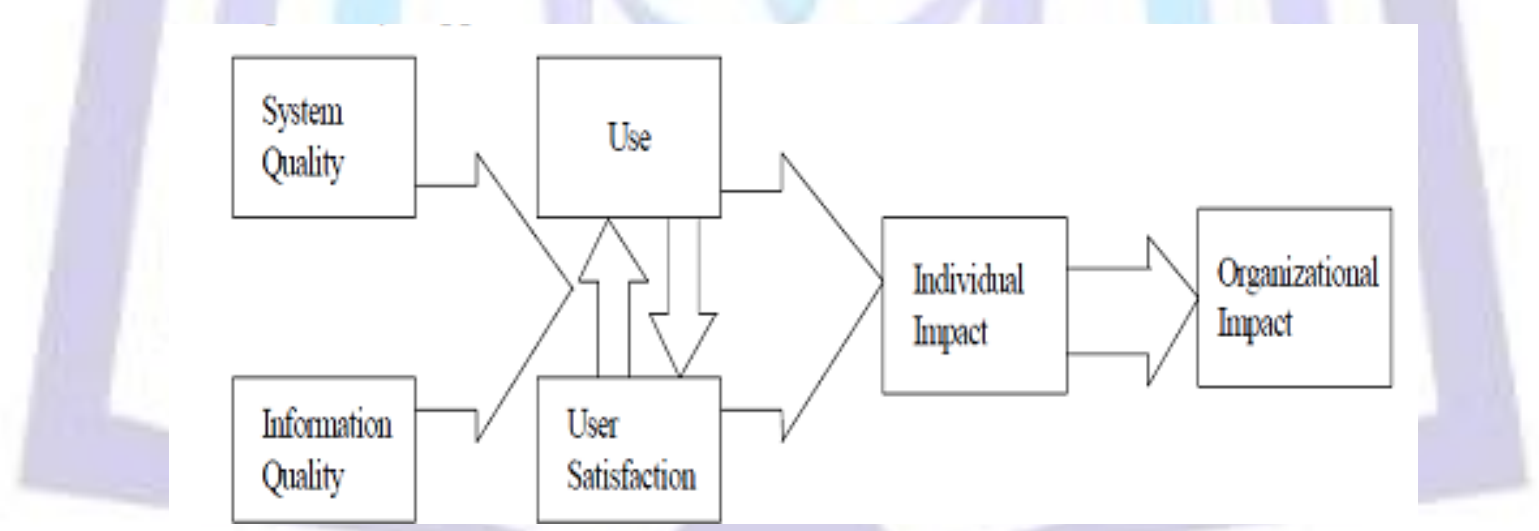

Figure 1: DeLone and McLean (1992) model of IS success

The contributions of this model were twofold in the field of IS success. First it categorized a large number of success measures into six main categories and secondly it suggested temporal and casual relationships between the constructs (Seddon,1997 ,McGill and Hobbs, 2003).A part of DeLone and McLean model was tested bySeddon and Kiew (1994). The use was replaced by usefulness and user involvement was added. The DeLone and McLean model was partially supported by their results. Seddon(1997)proposed another model which focused on casual relationships among the constructs.DeLone and McLean proposed a new model of IS success in the year 2003.DeLone\& McLean (2003) after reviewing the suggestions added a new construct "service quality" to the model and replaced all impact constructs with "net benefits". Figure 2 below shows the updated model. 


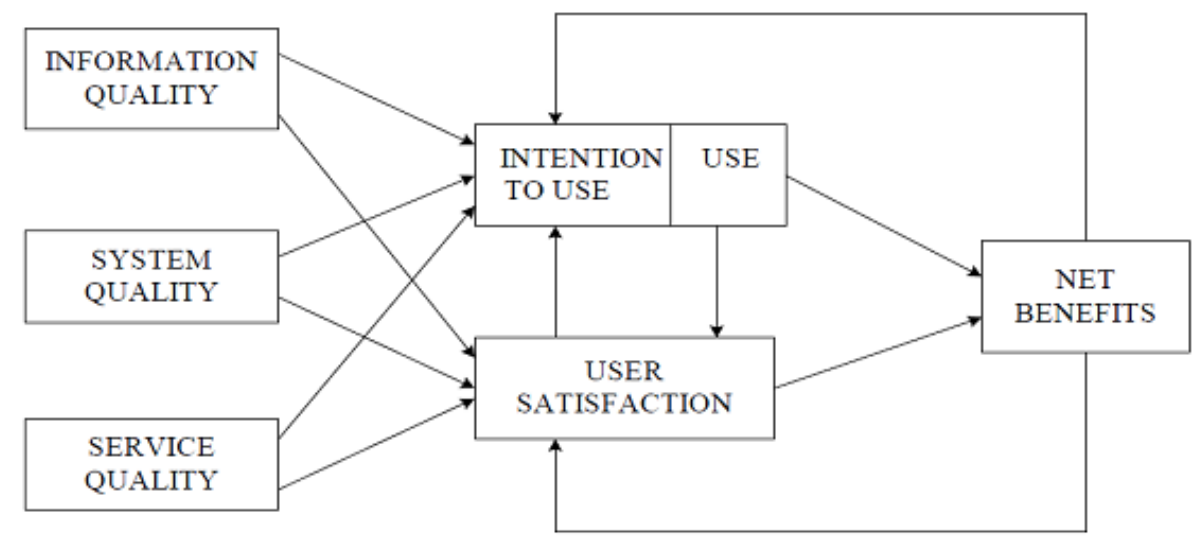

Figure 2: Updated DeLone and McLean IS success Model (2003)

\section{RESEARCH MODEL AND RESEARCH METHODOLOGY}

In this study the updated DeLone and McLean model is used to measure the success of DSS in banks of Oman. The research model is presented below in figure 3 .

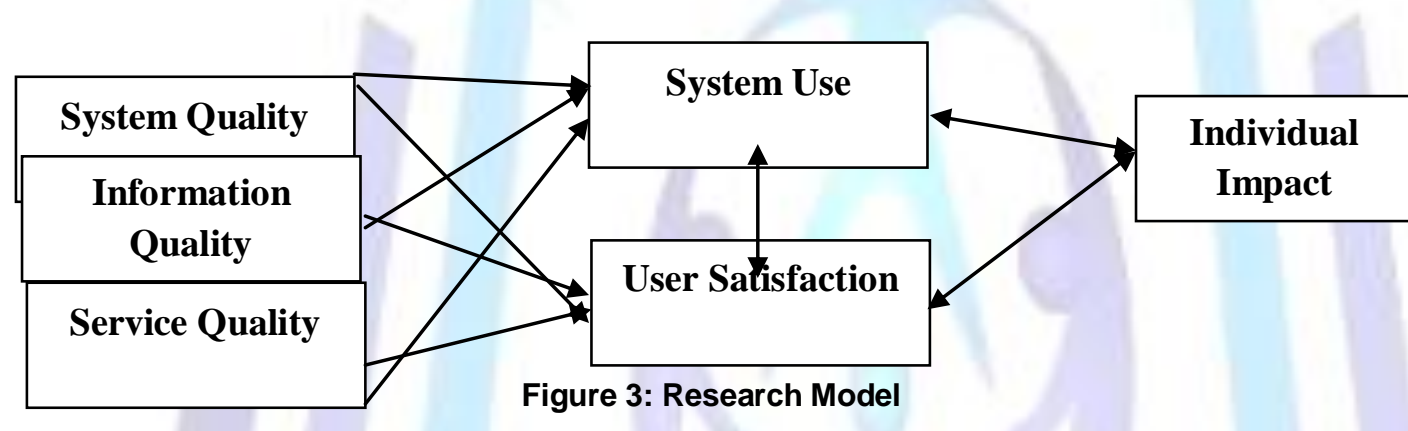

The main aim of this study is to validate the applicability of DeLone and McLean model in measuring DSS success in the banks of Oman. A questionnaire was used to collect data. Items for the constructs were taken from the studies done in the past. System quality and information quality were measured by Doll and Torkzadeh (1988) instrument with 12 items, service quality was measured by 22 items instrument from (Parasuraman, 1988), system use with 4 items by lgbaria et al.(1989), user satisfaction was measured bySeddon and Yip(1992) instrument containing four items and to measure individual impact instrument developed by Doll and Torkzadeh(1999) with 12 items was used. All responses were measured using Likert scales (1-5) ranging from "strongly agree" to "strongly disagree". Data was collected from the bank employees using decision support systems. A total of 405 questionnaires were distributed and 335 were collected out of which 28 questionnaires were incomplete. So the usable number of questionnaires was 307 . 
Table 1: Descriptive statistics of respondents

\begin{tabular}{|c|c|c|c|}
\hline & Characteristic & Number & Percentage \\
\hline \multirow[t]{2}{*}{ Gender } & male & 195 & 63.5 \\
\hline & female & 112 & 36.5 \\
\hline \multirow[t]{4}{*}{ Age } & $20-29$ & 23 & 7.5 \\
\hline & $30-39$ & 133 & 43.3 \\
\hline & $40-49$ & 95 & 30.9 \\
\hline & $>=50$ & 56 & 18.2 \\
\hline \multirow[t]{4}{*}{ Qualification } & High School & 8 & 2.6 \\
\hline & Diploma & 106 & 34.5 \\
\hline & Bachelor & 152 & 49.5 \\
\hline & Master & 41 & 13.4 \\
\hline \multirow{6}{*}{$\begin{array}{l}\text { Length of service in the } \\
\text { banking industry }\end{array}$} & $<1$ year & 1 & 3 \\
\hline & $1-5$ years & 37 & 12.1 \\
\hline & $6-10$ years & 128 & 41.7 \\
\hline & $11-15$ years & 82 & 26.7 \\
\hline & $16-20$ years & 39 & 12.7 \\
\hline & $>20$ years & 20 & 6.5 \\
\hline \multirow{6}{*}{$\begin{array}{l}\text { Length of service in the } \\
\text { current bank }\end{array}$} & $<1$ year & 4 & 1.3 \\
\hline & $1-5$ years & 76 & 22.8 \\
\hline & $6-10$ years & 141 & 45.9 \\
\hline & $11-15$ years & 67 & 21.8 \\
\hline & $16-20$ years & 20 & 6.5 \\
\hline & $>20$ years & 5 & 1.6 \\
\hline
\end{tabular}

\section{Source: Survey}

\section{DATA ANALYSIS}

The model was tested using smartPLS 2.0. SEM process is comprised of two steps. First step is to validate the measurement model and second includes assessment of the structural model. These steps are required to check the reliability and validity of the measures of all the constructs before drawing the final conclusions regarding their relationships (Barclay, Higgins, \& Thompson, 1995).

The model to be used in this study shows relationships from use to user satisfaction and vice versa, the relationship from use to individual impact and user satisfaction to individual impact. The updated D\&M model has bidirectional arrows between System use and User satisfaction as well as from System use tolndividual impact and User satisfaction to Individual impact. In SEM such relationships cannot be tested in the same model. So in this study four different structural models were created and tested. Model A depicts therelationship from system use to user satisfaction and from system use and user satisfaction to individual impact. Model B shows the relationship from user satisfaction to system use and from system use and user satisfaction to individual impact. Model $C$ depicts the relationship from system use to user satisfaction and from individual impact to system use and user satisfaction. Model D shows relationships from user satisfaction to system use and from individual impact to system use as well as user satisfaction.

\section{RESULTS}

\subsection{Measurement model}

The measurement model in PLS is evaluated by construct reliability, convergent reliability, discriminant validity and indicator reliability.

There are two measures to assess construct reliability: first is Cronbach's alpha and second is composite reliability (rc). According toNunnally (1978) value of 0.7 can be taken as a benchmark for 'modest' reliability. In this study all the 


\section{ISSN 2277-3061}

constructs were highly reliable. Values were close to 0.7 or higher than 0.7 for Cronbach's alpha as well as composite reliability.

Table 2,3,4 and 5 below show AVE, composite reliability, R square and Cronbach's alpha for all the four models.

Table 2: AVE, Composite reliability, R square, Cronbach's alpha: Measurement model A

\begin{tabular}{|c|c|c|c|c|}
\hline & AVE & $\begin{array}{c}\text { Composite } \\
\text { Reliability }\end{array}$ & R Square & Cronbachs Alpha \\
\hline Individual impact & 0.422698 & 0.896880 & 0.351970 & 0.875642 \\
\hline $\begin{array}{c}\text { Information } \\
\text { quality }\end{array}$ & 0.507474 & 0.860025 & & 0.804869 \\
\hline Service quality & 0.372478 & 0.928407 & & 0.919812 \\
\hline System quality & 0.464837 & 0.838014 & & 0.769814 \\
\hline System use & 0.307570 & 0.677554 & 0.045796 & 0.688265 \\
\hline User satisfaction & 0.670504 & 0.890341 & 0.236469 & 0.836014 \\
\hline
\end{tabular}

Table 3: AVE, Composite reliability, R square, Cronbach'salpha :Measurement model B

\begin{tabular}{|c|c|c|c|c|}
\hline & AVE & $\begin{array}{c}\text { Composite } \\
\text { Reliability }\end{array}$ & R Square & Cronbachs Alpha \\
\hline Individual impact & 0.422591 & 0.896828 & 0.350577 & 0.875642 \\
\hline $\begin{array}{c}\text { Information } \\
\text { quality }\end{array}$ & 0.507535 & 0.860074 & & 0.804869 \\
\hline Service quality & 0.372616 & 0.928450 & & 0.919812 \\
\hline System quality & 0.464805 & 0.838005 & & 0.769814 \\
\hline System use & 0.334187 & 0.736421 & 0.061631 & 0.688265 \\
\hline User satisfaction & 0.670444 & 0.890308 & 0.219787 & 0.836014 \\
\hline
\end{tabular}

Table 4: AVE, Composite reliability, R square, Cronbach's alpha- Measurement model C

\begin{tabular}{|c|c|c|c|c|}
\hline & AVE & $\begin{array}{c}\text { Composite } \\
\text { Reliability }\end{array}$ & R Square & Cronbachs Alpha \\
\hline Individual impact & 0.422396 & 0.896725 & & 0.875642 \\
\hline $\begin{array}{c}\text { Information } \\
\text { quality }\end{array}$ & 0.507530 & 0.860070 & & 0.804869 \\
\hline Service quality & 0.372622 & 0.928449 & & 0.919812 \\
\hline System quality & 0.464845 & 0.838018 & & 0.769814 \\
\hline System use & 0.327248 & 0.723219 & 0.038949 & 0.688265 \\
\hline User satisfaction & 0.670458 & 0.890309 & 0.393162 & 0.836014 \\
\hline
\end{tabular}


Table 5: AVE, Composite reliability, R square, Cronbach's alpha: Measurement model D

\begin{tabular}{|c|c|c|c|c|}
\hline & AVE & $\begin{array}{c}\text { Composite } \\
\text { Reliability }\end{array}$ & R Square & Cronbachs Alpha \\
\hline Individual impact & 0.422394 & 0.896724 & & 0.875642 \\
\hline $\begin{array}{c}\text { Information } \\
\text { quality }\end{array}$ & 0.507538 & 0.860078 & & 0.804869 \\
\hline Service quality & 0.372623 & 0.928452 & & 0.919812 \\
\hline System quality & 0.464826 & 0.838016 & & 0.769814 \\
\hline System use & 0.334271 & 0.736727 & 0.063512 & 0.688265 \\
\hline User satisfaction & 0.670351 & 0.890251 & 0.376223 & 0.836014 \\
\hline
\end{tabular}

According toFornell and Larcker (1981), convergent validity is assessed by average variance extracted(AVE) measure. AVE values should be more than 0.5(Segars,1997). In this study, AVE was close to 0.5 for some constructs and more than 0.5 for most of the constructs.

The discriminant validity can be assessed by calculating the square root of AVE of each construct (Fornell and Larcker,1981) and its value should be greater than other correlation values among the latent variables.

Tables6,7,8 and 9 show the values of discriminant validity calculated according to Fornell and Larcker criteria.

Table 6: Discriminant validity Fornell-Larcker Criterion for measurement model A

\begin{tabular}{|c|c|c|c|c|c|c|}
\hline & $\begin{array}{c}\text { Individual } \\
\text { impact }\end{array}$ & $\begin{array}{c}\text { Information } \\
\text { quality }\end{array}$ & $\begin{array}{c}\text { Service } \\
\text { quality }\end{array}$ & $\begin{array}{c}\text { System } \\
\text { quality }\end{array}$ & $\begin{array}{c}\text { System } \\
\text { use }\end{array}$ & $\begin{array}{c}\text { User } \\
\text { satisfaction }\end{array}$ \\
\hline $\begin{array}{c}\text { Individual } \\
\text { impact }\end{array}$ & $\mathbf{0 . 6 5 0 1 5 2}$ & & & & & \\
\hline $\begin{array}{c}\text { Informati } \\
\text { on quality }\end{array}$ & 0.478780 & $\mathbf{0 . 7 1 2 3 7 2}$ & & & & \\
\hline $\begin{array}{c}\text { Service } \\
\text { quality }\end{array}$ & 0.411261 & 0.386468 & $\mathbf{0 . 6 1 0 3 0 9}$ & & & \\
\hline $\begin{array}{c}\text { System } \\
\text { quality }\end{array}$ & 0.469508 & 0.578123 & 0.364460 & $\mathbf{0 . 6 8 1 7 8 9}$ & & \\
\hline $\begin{array}{c}\text { System } \\
\text { use }\end{array}$ & 0.054071 & 0.095822 & 0.185086 & -0.003527 & $\mathbf{0 . 5 5 4 5 8 9}$ & \\
\hline $\begin{array}{c}\text { User } \\
\text { satisfacti } \\
\text { on }\end{array}$ & 0.584311 & 0.433650 & 0.264167 & 0.382361 & -0.082647 & $\mathbf{0 . 8 1 8 8 4 3}$ \\
\hline
\end{tabular}

Note: Values are represented in bold letters.

Table 7 :Discriminant validity Fornell-Larcker Criterion for measurement model B

\begin{tabular}{|c|c|c|c|c|c|c|}
\hline & $\begin{array}{c}\text { Individual } \\
\text { impact }\end{array}$ & $\begin{array}{c}\text { Information } \\
\text { quality }\end{array}$ & $\begin{array}{c}\text { Service } \\
\text { quality }\end{array}$ & $\begin{array}{c}\text { System } \\
\text { quality }\end{array}$ & $\begin{array}{c}\text { System } \\
\text { use }\end{array}$ & $\begin{array}{c}\text { User } \\
\text { satisfaction }\end{array}$ \\
\hline $\begin{array}{c}\text { Individual } \\
\text { impact }\end{array}$ & $\mathbf{0 . 6 5 0 0 7}$ & & & & & \\
\hline $\begin{array}{c}\text { Informati } \\
\text { on quality }\end{array}$ & 0.478983 & $\mathbf{0 . 7 1 2 4 1 5}$ & & & & \\
\hline $\begin{array}{c}\text { Service } \\
\text { quality }\end{array}$ & 0.410655 & 0.387557 & $\mathbf{0 . 6 1 0 4 2 3}$ & & & \\
\hline
\end{tabular}




\begin{tabular}{|c|c|c|c|c|c|c|}
\hline $\begin{array}{c}\text { System } \\
\text { quality }\end{array}$ & 0.469519 & 0.578041 & 0.364811 & $\mathbf{0 . 6 8 1 7 6 6}$ & & \\
\hline $\begin{array}{c}\text { System } \\
\text { use }\end{array}$ & 0.031100 & 0.096725 & 0.156961 & 0.001947 & $\mathbf{0 . 5 7 8 0 8 9}$ & \\
\hline $\begin{array}{c}\text { User } \\
\text { satisfacti } \\
\text { on }\end{array}$ & 0.584930 & 0.433372 & 0.264569 & 0.382652 & -0.103001 & $\mathbf{0 . 8 1 8 8 0 6}$ \\
\hline
\end{tabular}

Note: Values are represented in bold letters.

Table 8: Discriminant validity Fornell-Larcker Criterion for measurement model C

\begin{tabular}{|c|c|c|c|c|c|c|}
\hline & $\begin{array}{c}\text { Individual } \\
\text { impact }\end{array}$ & $\begin{array}{c}\text { Information } \\
\text { quality }\end{array}$ & $\begin{array}{l}\text { Service } \\
\text { quality }\end{array}$ & $\begin{array}{l}\text { System } \\
\text { quality }\end{array}$ & $\begin{array}{l}\text { System } \\
\text { use }\end{array}$ & $\begin{array}{c}\text { User } \\
\text { satisfaction }\end{array}$ \\
\hline $\begin{array}{c}\text { Individual } \\
\text { impact }\end{array}$ & $\begin{array}{c}0.64991999 \\
5\end{array}$ & & & & & \\
\hline $\begin{array}{l}\text { Informati } \\
\text { on quality }\end{array}$ & 0.478892 & $\begin{array}{c}0.71241139 \\
8\end{array}$ & & & & \\
\hline $\begin{array}{l}\text { Service } \\
\text { quality }\end{array}$ & 0.409490 & 0.387205 & $\begin{array}{c}0.6104277 \\
19\end{array}$ & & & \\
\hline $\begin{array}{l}\text { System } \\
\text { quality }\end{array}$ & 0.469257 & 0.577928 & 0.364669 & $\begin{array}{c}0.681795 \\
424\end{array}$ & & \\
\hline $\begin{array}{l}\text { System } \\
\text { use }\end{array}$ & 0.026643 & 0.091744 & 0.164846 & $\begin{array}{c}- \\
0.002728\end{array}$ & $\begin{array}{c}0.5720559 \\
41\end{array}$ & \\
\hline $\begin{array}{c}\text { User } \\
\text { satisfacti } \\
\text { on }\end{array}$ & 0.585519 & 0.433164 & 0.263967 & 0.382489 & -0.104223 & $\begin{array}{c}0.81881499 \\
7\end{array}$ \\
\hline
\end{tabular}

Note: Values are represented in bold letters.

Table 9: Discriminant validity Fornell-Larcker Criterion for measurement model D

\begin{tabular}{|c|c|c|c|c|c|c|}
\hline & $\begin{array}{c}\text { Individual } \\
\text { impact }\end{array}$ & $\begin{array}{c}\text { Informatio } \\
\text { n quality }\end{array}$ & $\begin{array}{l}\text { Service } \\
\text { quality }\end{array}$ & $\begin{array}{l}\text { System } \\
\text { quality }\end{array}$ & $\begin{array}{l}\text { System } \\
\text { use }\end{array}$ & $\begin{array}{c}\text { User } \\
\text { satisfaction }\end{array}$ \\
\hline $\begin{array}{c}\text { Individual } \\
\text { impact }\end{array}$ & $\begin{array}{c}0.64991845 \\
6\end{array}$ & & & & & \\
\hline $\begin{array}{c}\text { Informatio } \\
\text { n quality }\end{array}$ & 0.479006 & $\begin{array}{c}0.71241701 \\
3\end{array}$ & 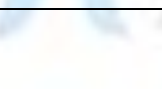 & & & \\
\hline $\begin{array}{l}\text { Service } \\
\text { quality }\end{array}$ & 0.409624 & 0.387676 & $\begin{array}{c}0.6104285 \\
38\end{array}$ & & & \\
\hline $\begin{array}{l}\text { System } \\
\text { quality }\end{array}$ & 0.469316 & 0.578032 & 0.364864 & $\begin{array}{c}0.68178 \\
149\end{array}$ & & \\
\hline $\begin{array}{l}\text { System } \\
\text { use }\end{array}$ & 0.029720 & 0.096032 & 0.155155 & $\begin{array}{c}0.00135 \\
7\end{array}$ & $\begin{array}{c}0.5781617 \\
42\end{array}$ & \\
\hline $\begin{array}{c}\text { User } \\
\text { satisfactio } \\
n\end{array}$ & 0.585885 & 0.433084 & 0.264308 & $\begin{array}{c}0.38291 \\
7\end{array}$ & -0.105218 & $\begin{array}{c}0.81874965 \\
6\end{array}$ \\
\hline
\end{tabular}

Note: Values are represented in bold letters.

The results presented in all the four tables confirm that all diagonal elements are having values greater than the offdiagonal elements in the respective row and column.

Indicator reliability is calculated by finding the square of outer loadings. According to Hulland (1999) value 0.4 or higher are acceptable and greater than 0.7 is considered good.

In this study most of the items exhibited indicator reliability as values were close to 0.4 for some and higher than 0.4 for most of the items. 


\subsection{Structural model}

After getting satisfactory results for the measurement model for all the four models bootstrapping was performed for all the four models. Results for path coefficients of model A are presented in table 10 below.

Table 10 :Results from T-Statistics of Path Coefficients for Model A

\begin{tabular}{|c|c|c|}
\hline & T-Statistics & Results \\
\hline Information quality -> System use & 0.800462 & Not supported \\
\hline Information quality -> User satisfaction & 4.190463 & Not supported \\
\hline Service quality -> System use & 1.056346 & Not supported \\
\hline Service quality $->$ User satisfaction & 1.887308 & Not supported \\
\hline System quality -> System use & 1.054996 & supported \\
\hline System quality $->$ User satisfaction & 2.353406 & Not supported \\
\hline System use $->$ Individual impact & 1.065108 & Not supported \\
\hline System use $->$ User satisfaction & 1.519518 & Strongly supported \\
\hline User satisfaction $->$ Individual impact & 10.201357 & \\
\hline
\end{tabular}

Note: for a 2-tailed t-test at a significance level of 5\%, t-statistics value should be greater than 1.96 for the path coefficient to be significant.

Results for path coefficients of model B are presented in table 11 below.

Table 11 :Results from T-Statistics of Path Coefficients for Model B

\begin{tabular}{|c|c|c|}
\hline & T Statistics & Results \\
\hline Information quality -> System use & 1.490731 & Not supported \\
\hline $\begin{array}{c}\text { Information quality -> User } \\
\text { satisfaction }\end{array}$ & 4.020682 & Strongly supported \\
\hline Service quality $->$ System use & 1.102691 & Not supported \\
\hline Service quality $->$ User satisfaction & 1.395625 & Not supported \\
\hline System quality -> System use & 0.746965 & Not supported \\
\hline System quality -> User satisfaction & 2.510542 & supported \\
\hline System use -> Individual impact & 1.071033 & Not supported \\
\hline $\begin{array}{c}\text { User satisfaction -> Individual } \\
\text { impact }\end{array}$ & 10.178323 & Strongly supported \\
\hline User satisfaction -> System use & 1.945359 & Not supported \\
\hline
\end{tabular}

Results for path coefficients of model $\mathrm{C}$ are presented in table 12 below. 
Table 12: Results from T-Statistics of Path Coefficients for Model C

\begin{tabular}{|c|c|c|}
\hline & T Statistics & Results \\
\hline $\begin{array}{c}\text { Individual impact -> System use } \\
\text { satisfaction }\end{array}$ & 0.369137 & Not supported \\
\hline $\begin{array}{c}\text { Individual impact -> User } \\
\text { Information quality -> System use } \\
\text { Information quality -> User } \\
\text { satisfaction }\end{array}$ & 4.945859 & Not supported \\
\hline $\begin{array}{c}\text { Service quality }->\text { System use } \\
\text { Service quality }->\text { User satisfaction }\end{array}$ & 2.872756 & Nupported \\
\hline System quality $->$ System use & 1.100613 & Not supported \\
\hline System quality $->$ User satisfaction & 0.002631 & Not supported \\
\hline System use -> User satisfaction & 1.828432 & Not supported \\
\hline
\end{tabular}

Results for path coefficients of model $D$ are presented in table 13 below.

Table 13 :Results from T-Statistics of Path Coefficients for Model D

\begin{tabular}{|c|c|c|}
\hline \multicolumn{1}{|c|}{ T Statistics } & Results \\
\hline $\begin{array}{c}\text { Individual impact -> System use } \\
\text { satisfaction }\end{array}$ & 0.421105 & Strongly supported \\
\hline Individual impact -> User & 4.868148 & Not supported \\
\hline Information quality -> System use & 1.458524 & Supported \\
\hline Infarmation quality -> User & 2.625439 & Not supported \\
\hline Service quality -> System use & & Not supported \\
\hline Service quality -> User satisfaction & 0.482702 & Not supported \\
\hline System quality -> System use & 0.735821 & Not supported \\
\hline User satisfaction -> System use & 1.936115 & \\
\hline
\end{tabular}

T-statistics values higher than 1.96 at $5 \%$ significance level are considered significant. Results of t-statistics from table 10 and 11 suggest that the relationship between system quality to user satisfaction, information quality to user satisfaction and user satisfaction to individual impact are significant. All other relationships were found non-significant.

Similarly results from table 12 and 13show that information quality to user satisfaction and from individual impact to user satisfaction are significant.

Based upon the results obtained from these four models the final model was derived. The bidirectional relationship between user satisfaction and individual impact could not be tested at once. So it was run twice. The diagrams along with their outputs are represented below. 


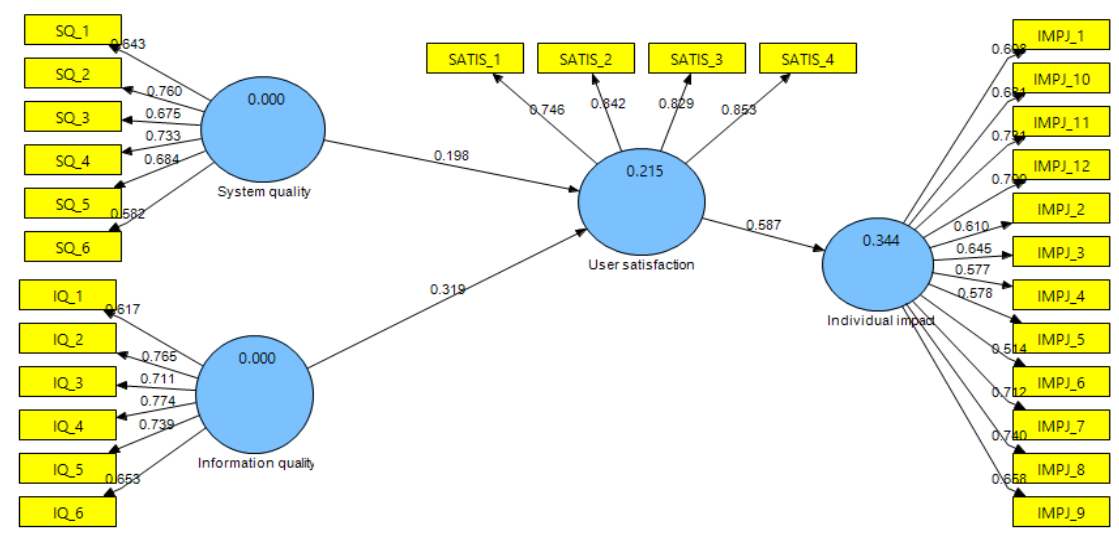

Figure 4: Final model showing relationship from user satisfaction to individual impact

Table 14: AVE, Composite reliability, $R$ square, Cronbach's alpha

\begin{tabular}{|c|c|c|c|c|}
\hline & AVE & Composite Reliability & R Square & Cronbachs Alpha \\
\hline $\begin{array}{c}\text { Individual } \\
\text { impact }\end{array}$ & 0.422285 & 0.896669 & 0.343989 & 0.875642 \\
\hline $\begin{array}{c}\text { Information } \\
\text { quality }\end{array}$ & 0.507353 & 0.859920 & & 0.804869 \\
\hline System quality & 0.464823 & 0.838017 & & 0.769814 \\
\hline $\begin{array}{c}\text { User } \\
\text { satisfaction }\end{array}$ & 0.670274 & 0.890223 & 0.214738 & 0.836014 \\
\hline
\end{tabular}

Table 14 shows that values of Cronbach's alpha and composite reliability are higher than 0.7 so it can be concluded that all the constructs are reliable.

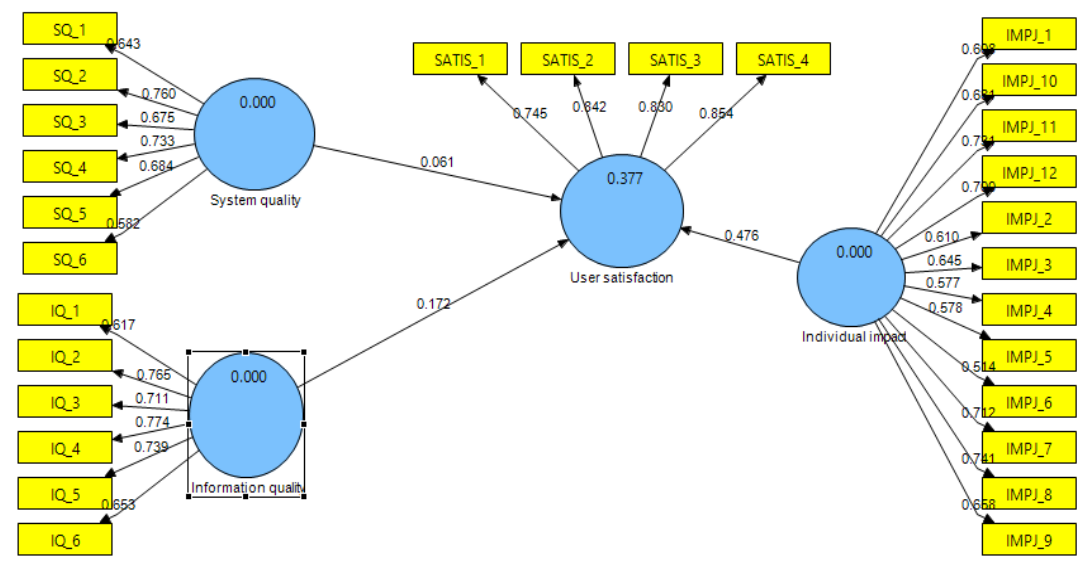

Figure 5: Final model showing relationship from individual impact to user satisfaction 
Table 15: AVE, Composite reliability, $R$ square, Cronbach's alpha

\begin{tabular}{|c|c|c|c|c|}
\hline & AVE & Composite Reliability & R Square & Cronbachs Alpha \\
\hline Individual impact & 0.422285 & 0.896668 & & 0.875642 \\
\hline Information quality & 0.507354 & 0.859921 & & 0.804869 \\
\hline System quality & 0.464826 & 0.838018 & & 0.769814 \\
\hline User satisfaction & 0.670280 & 0.890224 & 0.376899 & 0.836014 \\
\hline
\end{tabular}

Table 15 shows that values of Cronbach's alpha and composite reliability are higher than 0.7 so it can be concluded that all the constructs are reliable.

\section{DISCUSSION}

SEM was run four times to test the bidirectional relationships from system use to user satisfaction, system use to individual impact and from user satisfaction to individual impact. In analysis many of the relationships were found insignificant. Most of the insignificant relationships were related to two constructs-service quality and system use. The reason for this could be that SEM could not represent all bidirectional relationships in one model and it might have led to the specification error.

The results also showed that system quality and information quality were mainly leading to user satisfaction which in turn was influencing individual impact whereas service quality was found to be insignificant. All indicators towards system use were insignificant.

So the banks should focus more on system quality and information quality of the decision support systems. This will lead to the success of these systems.

\section{CONCLUSION}

Validity of DeLone and McLean model of information system was examined in this study in DSS context. Results showed that many of the relationships in the model were not significant. Service quality and system use were found to be the least influential among all. Information quality and system quality influenced user satisfaction. User satisfaction influenced individual impact. Individual impact was also found to influence user satisfaction.

The main limitation of this study was that bidirectional relationship from system use to user satisfaction, from system use to individual impact and from user satisfaction to individual impact could not be measured at one structural equation modelling analysis.

\section{REFERENCES}

[1] Barclay, D., Higgins, C., Thompson, R. 1995. The partial least squares (PLS) approach to causal modelling: Personal computer adoption and use as an illustration. Technology Studies, vol. 2 , no. 2, pp. 285-309.

[2] Brown, I. and Jayakody, R.2008. B2C e-Commerce Success: a Test and Validation of a revised conceptual model. The electronic journal of infromation systems evaluation, vol. 11, no. 3, pp. 167-184.

[3] DeLone W.H. andMcLean E.R. 1992. Information systems success: The quest for the dependent variable. Information Systems Research, vol. 3, no. 1, pp.60-95.

[4] DeLone W.H. and McLean E.R. 2003. The DeLone and McLean model of information systems success: A ten-year update. Journal of Information systems Management, vol. 4, no. 19, pp. 9-26.

[5] Doll, William J., and GholamrezaTorkzadeh. 1988. The Measurement of End-user Computing Satisfaction. MIS Quarterly, vol. 12, no. 2,pp. 258-274.

[6] Fornell, Claes, and David F. Larcker. 1981. Evaluating structural equation models with unobservable variables and measurement error. Journal of Marketing Research, vol. 18, no. 1, pp. 39-50.

[7] Hulland, John. 1999. Use of partial least squares (PLS) in strategic management research: A review of four recent studies. Strategic Management Journal, vol. 20, no. 2, pp. 195-204.

[8] Igbaria, M., Pavri, F., \& Huff, S. 1989. Microcomputer applications. An empirical look at usage. Information \& Management,vol. 16,no. 4, pp.187-196.

[9] Little, John D. C.1970. Models and Managers: The Concept of a Decision Calculus. Management Science, vol.16, no. 8, B466-485.

[10] Nunnally, J. 1978. Psychometric Theory (second ed.). New York: McGraw Hill. 


\section{ISSN 2277-3061}

[11] Parasuraman, A. 1988. SERVQUAL: A multiple item scale for measuring consumer perceptions of service quality. Journal of Retailing, vol. 64, no. 1, pp.12-40.

[12] Seddon, P.B., and Yip, S. K. 1992. An empirical evaluation of User Information Satisfaction (UIS) Measures for Use with General Ledger Account Software. Journal of Information Systems,pp.75-92.

[13] Seddon, P.B., and Kiew, M.-Y. 1994. A Partial Test and Development of the DeLone and McLean Model of Is Success. Proceedings of the 15th International Conference on Information Systems (ICIS 94). Vancouver, Canada: pp. 99-110.

[14] Seddon, P. B. 1997. A respecification and extension of the DeLone and McLean model of IS success. Information Systems Research, vol. 8, no. 3,pp. 240-253.

[15]Segars, A. H. 1997. Assessing the unidimensionality of measurement: A paradigm and illustration within the context of information systems research. Omega,vol. 25, no. 1, pp.107--121.

[16]Tanya, McGill, Hobbs Valerie, and Klobas Jane. 2003. User developed applications and information systems success: A test of DeLone and McLean's model. Information Resources Management Journal, vol. 16, no. 1,pp. 24-45.

[17] Torkzadeh, G., and W. J. Doll. 1999. The development of a tool for measuring the perceived impact of information technology on work. Omega, International journal of management science, vol. 27, no. 3,pp. 327-339. 